\title{
Multi-AntenNa Localization Method
}

\author{
John R Rankin \\ Federation University Australia
}

\begin{abstract}
In a dense wireless network where sensor nodes run on batteries it is essential for the continued running of the network, to be able to accurately locate and identify nodes that need battery replacement. Accurate localization of nodes is also necessary for the correlation of sensed data with position in the environment covered by the network. In this paper a new approach to node localization which is fine grained, rangebased, anchor free and GPS free, is presented which is cheap in hardware components and cheap in software computation. It concerns the localization of cluster nodes relative to the cluster head and is suitable for cases of stationary or slow moving nodes. The method is demonstrated for a simple one-level wireless network.
\end{abstract}

\section{KEYWORDS}

WSN (Wireless Sensor Network), sensor node, RSSI, localization.

\section{INTRODUCTION}

The problem of determining the position of radio sources relative to a given detector location is a common problem for which several solutions have been proposed and are also in use. The five most commonly used methods are Lateration, Angulation, Trilateration, Multilateration and Triangulation [1]. Each of these methods is well described in the review article [1]. Lateration is based on distance measurements from the inverse square law for EMR signal intensity as are likewise Trilateration and Multilateration. The distance between sites is determined by RSSI measurements on the assumption that the signal intensity at the source is a known constant. In another work [2] a method is given for computing the positions of sites when the distance matrix (giving the distances between all sites) is known. Triangulation on the other hand involves the use of directional antennas at points $\mathrm{A}$ and $\mathrm{B}$ which each point to the radio source at $\mathrm{C}$ the third point of a triangle whose position is then computed trigonometrically. Angulation also uses directional signal detection to obtain angles to the source which again can be used to compute relative positions in 2D or 3D. Directional antennas make more efficient use of battery energy [5] but are not applicable to networks with randomly placed nodes and dynamic connectivity architectures. In general the use of directional antennas needs the direction of the antenna to rotate about a vertical axis to sweep around the horizontal plane at constant speed similar to the use of naval radar systems to search for input signals. However sweeping directional antennas contain mechanical parts that wear out, consume battery power and require regular maintenance. Mechanical moving parts are ultimately more expensive in maintenance and not suitable for the field placement of the wireless nodes of a WSN. Other more complex designs for directional antennas using electronic switching rather than moving parts have been considered $[3,4,5,7]$ and these require additional circuitry and node battery power consumption. While a wide variety of antenna types exist that may be used in WSNs [3,6] few of the standard types of antenna are simple, small, low cost and low power consumptive enough to be suitable [1]. A simpler approach for determining the location of radio sources in the 2D plane using only omnidirectional antennas and low battery power consumption is presented in this work. 


\section{Distance Measurement With An Omni directional Antenna}

When a wireless node is transmitting data its antenna produces a signal strength of EMR (ElectroMagnetic Radiation) which drops off according to the inverse square law. In suitable units for signal strength, this law is:

$$
\psi(r)=\frac{\psi_{0}}{r^{2}}
$$

where $\mathrm{r}$ is the horizontal distance from the node and $\psi_{0}$ is the signal strength produced by the node at its antenna which corresponds to $\mathrm{r}=1$ in suitable distance units. When a source is not transmitting $\psi_{0}=0$ so then $\psi(r)=0$ and no signal is detectable. For $\mathrm{r}<1$ we can assume that $\psi(r)=\psi_{0}$ and as such no anomalous infinities will occur.

An RSSI or signal strength detector is an antenna connected to an amplifier and then fed into an ADC (analog to digital converter) chip. If the characteristic minimal signal strength differential detectable by the detector is $\Delta \psi$ then the accuracy of the detector or minimum distinguishable distance measurable is given by

$$
\Delta r=\frac{1}{2 \psi_{0}} r^{3} \Delta \psi
$$

This shows that the error in the distance measurement $\Delta r$ increases with the cube of the distance from the source but that the error is less for stronger sources. For the accurate positioning of radio sources we therefore need $\mathrm{r}$ and $\Delta \psi$ small and $\psi_{0}$ large. The quantity $\Delta \psi$ is however a fixed characteristic of the detector as provided by the manufacturer and so cannot be varied. In the following discussions it will be assumed that the scale of the network $\mathrm{R}$ is much larger than $\Delta r$ so that localization will be accurate. The radius of radio reach $\rho$ of a source occurs at the distance

$$
\text { where } \psi(\rho)=\Delta \psi \text {. Therefore } \rho=\sqrt{\frac{\psi_{0}}{\Delta \psi}} \text {. }
$$

\section{LOCALIZING IN 2D USING OMNIDIRECTIONAL ANTENNAS}

Suppose that node A located at the origin in 2D has $\mathrm{n}$ fixed omnidirectional antennas for detecting signal strength at known positions $A_{i}$ relative to $A$ in $2 \mathrm{D}$ for $\mathrm{i}=1$ to $\mathrm{n}$. Suppose that node $\mathrm{A}$ is required to detect the position of source node $\mathrm{S}$ which is within wireless range $\rho$ of node $\mathrm{A}$. Denote the signal strength of node $\mathrm{S}$ detected at each detector antenna $\mathrm{A}_{\mathrm{i}}$ by $\psi_{i}$. Then the distance of node $S$ from each detector antenna $A_{i}$ is given by

$$
\begin{gathered}
r_{i}=\sqrt{\frac{\psi_{0}}{\psi_{i}}} \\
\left(x_{S}-A_{i x}\right)^{2}+\left(y_{S}-A_{i y}\right)^{2}=r_{i}^{2}
\end{gathered}
$$

for $\mathrm{i}=1$ to $\mathrm{n}$. Subtracting equation 1 from equations 2 to $\mathrm{n}$ gives $\mathrm{n}-1$ linear equations in 2 unknowns:

$$
\left(A_{i x}-A_{1 x}\right) x_{S}+\left(A_{i y}-A_{1 y}\right) y_{S}=\frac{1}{2}\left\{A_{i x}{ }^{2}-A_{1 x}{ }^{2}+A_{i y}{ }^{2}-A_{1 y}{ }^{2}+r_{1}{ }^{2}-r_{i}{ }^{2}\right\}
$$


Since we have two unknowns, $x_{S}$ and $y_{S}$, it follows that a minimum of $n=3$ detecting antennas is sufficient to accurately pinpoint the location $\left(\mathrm{x}_{\mathrm{S}}, \mathrm{y}_{\mathrm{S}}\right)$ of any source $\mathrm{S}$. The position of the radio source for $\mathrm{n}=3$ is then given by

$$
\begin{aligned}
& D=\left(A_{2 x}-A_{1 x}\right)\left(A_{3 y}-A_{1 y}\right)-\left(A_{3 x}-A_{1 x}\right)\left(A_{2 y}-A_{1 y}\right) \\
& D_{x 1}=\left\{A_{2 x}{ }^{2}-A_{1 x}{ }^{2}+A_{2 y}{ }^{2}-A_{1 y}{ }^{2}+r_{1}{ }^{2}-r_{2}{ }^{2}\right\}\left(A_{3 y}-A_{1 y}\right) \\
& D_{x 2}=\left\{A_{3 x}{ }^{2}-A_{1 x}{ }^{2}+A_{3 y}{ }^{2}-A_{1 y}{ }^{2}+r_{1}{ }^{2}-r_{3}{ }^{2}\right\}\left(A_{2 y}-A_{1 y}\right) \\
& D_{y 1}=\left(A_{2 x}-A_{1 x}\right)\left\{A_{3 x}{ }^{2}-A_{1 x}{ }^{2}+A_{3 y}{ }^{2}-A_{1 y}{ }^{2}+r_{1}{ }^{2}-r_{3}{ }^{2}\right\} \\
& D_{y 2}=\left(A_{3 x}-A_{1 x}\right)\left\{A_{2 x}{ }^{2}-A_{1 x}{ }^{2}+A_{2 y}{ }^{2}-A_{1 y}{ }^{2}+r_{1}{ }^{2}-r_{2}{ }^{2}\right\} \\
& x_{S}=\frac{D_{x 1}-D_{x 2}}{2 D} \\
& y_{S}=\frac{D_{y 1}-D_{y 2}}{2 D}
\end{aligned}
$$

Such a setup of 3 omnidirectional antennas for a node is herein termed a localizator. The raw data from the localizator is $\left\{\psi_{1}, \psi_{2}, \psi_{3}\right\}$ which must be converted to $\left\{r_{1}, r_{2}, r_{3}\right\}$ and all other parameters in the above equations are known constants. It is assumed that the three antennas, $A_{1}$, $A_{2}$ and $A_{3}$, are set at the vertices of an equilateral triangle by the manufacturer and that the localizator is placed with the orientation relative to the $\mathrm{x}$ and $\mathrm{y}$ axes as shown in figure 1 . However it does not matter whether the antennas $A_{1}, A_{2}$ and $A_{3}$ are rotated about $A$ by any angle so long as this angle remains constant: the effect is only a rotation of the coordinate system and of the local $\mathrm{x}$ and $\mathrm{y}$ axes relative to point $\mathrm{A}$. In the frame of the localizator therefore the constants in the above equations are

$$
\begin{aligned}
& A_{1 x}=\frac{\sqrt{3}}{2} b, A_{1 y}=-\frac{1}{2} b \\
& A_{2 x}=0, A_{2 y}=b \\
& A_{3 x}=-\frac{\sqrt{3}}{2} b, A_{3 y}=-\frac{1}{2} b
\end{aligned}
$$

where $b$ is the distance of any antenna from the centre of the node as set by the manufacturer. This then assumes that we try to orient all placed nodes that have associated localizators such that $\mathrm{A}_{2}$ always points to a given fixed distant landmark which determines the y direction in the plane. (Of course we could have alternatively pointed $\mathrm{A}_{1}$ along the $\mathrm{x}$ axis and oriented all localizators

with $\mathrm{A}_{1}$ pointing to a specific distant landmark indicating the $\mathrm{x}$ direction in the $2 \mathrm{D}$ plane and the triangle in Figure 1 would be rotated anticlockwise by 30 degrees.) 


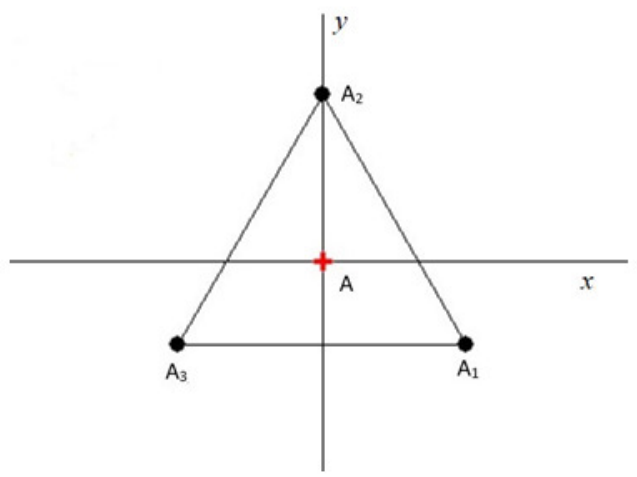

Figure 1 showing the positions of the antennas at $A_{1}, A_{2}$ and $A_{3}$ relative to the node at $A$.

\section{ApPlication WSN}

In this work we are concerned with a WSN which is structured as a mains powered base station wirelessly connected to a number N1 of clusters (or "subnets"). The whole WSN is considered to lie in a plane. Each cluster consists of an aggregator node as cluster head and N2 sensor nodes surrounding the aggregator node [8]. The sensor nodes in a cluster send data to the aggregator node of that cluster and the aggregator node relays the data on to the base station. For example in the case of a subnet consisting of $\mathrm{N} 2=4$ sensor nodes, A, B, C and D, they will be arranged roughly at the corners of a square of side length a and the aggregator node $\mathrm{E}$ is roughly at the centre of the square and we want to establish accurate positioning of these nodes. This is the process of localization. The base station should initiate the localization process and thereby tell all nodes of the WSN to cease transmissions resulting in radio silence except for commands from the base station. The base station then sends a signal to the first aggregator node telling it to localize all N2 sensor nodes in its cluster. The aggregator node, say node E, is assumed to be at the origin of $2 \mathrm{D}$ coordinates for its cluster. To do the localization task, node $\mathrm{E}$ sends a radio signal to tell all its sensor nodes A, B, C ... to cease transmissions until localization is finalized and then node $\mathrm{E}$ asks each sensor node in its cluster in turn to transmit long enough for node $\mathrm{E}$ to locate it using the localizator at $\mathrm{E}$. Once all locations relative to $\mathrm{E}$ are known to $\mathrm{E}$, then node $\mathrm{E}$ sends the data to the base station. The base station also has a localizator and uses its own multiantenna RSSI detector to determine the coordinates of $\mathrm{E}$ relative to the base station. The base station now has the coordinates of $\mathrm{E}$ and the sensors $\mathrm{A}, \mathrm{B}, \mathrm{C} \ldots$ of the $\mathrm{E}$ subnet and then proceeds to repeat this process for the second aggregator node in the WSN and so on. Once all nodes of the WSN are located relative to the base station, the sensor nodes can return to their normal operational transmission mode. Of course any node, in a subnet could do the localization process but it is convenient if the task is undertaken by the aggregator for then only aggregator nodes and not sensor nodes need to have multi-antenna RSSI detectors to save costs. If the aggregator nodes are not all exactly oriented with their three antennas in the same orientations as the base station then an adjustment in the code of the base station can compensate for this. To achieve this, each aggregator should not only localize the sensor nodes of its own subnet, but it should also localize the base station relative to the aggregator's rotated coordinate system. When the aggregator sends the base station the coordinates of the base station relative to the

aggregator, the base station can compare these coordinates with its own determination of the aggregator's coordinates relative to the base station. Let the coordinates of the aggregator relative to the base station be $\vec{u}^{\prime}=\left(u_{x}^{\prime}, u_{y}^{\prime}\right)$ and the coordinates of the base station relative to the 
aggregator in the aggregator's rotated frame be $\vec{u}=\left(u_{x}, u_{y}\right)$. If the aggregator's frame of reference is un-rotated relative to the base station's frame of reference then $\vec{u}^{\prime}=-\vec{u}$ otherwise the base station computes the angle between the two vectors by

$\theta=\pi-\cos ^{-1}\left(\frac{u_{x} u_{x}^{\prime}+u_{y} u_{y}^{\prime}}{r^{2}}\right)$

where $\mathrm{r}$ is the distance between the base station and the aggregator (according to either localizator). The coordinates of any node A of the aggregator's subnet in the base station's frame of reference is therefore:

$$
\begin{aligned}
& A_{x}^{\prime}=E_{x}{ }_{x}+A_{x} \cos \theta-A_{y} \sin \theta \\
& A_{y}^{\prime}{ }_{y}=E^{\prime}{ }_{y}+A_{x} \sin \theta+A_{y} \cos \theta
\end{aligned}
$$

where $\left(\mathrm{A}_{\mathrm{x}}, \mathrm{A}_{\mathrm{y}}\right)$ are the coordinates of $\mathrm{A}$ relative to the aggregator $\mathrm{E}$ and $\left(\mathrm{E}_{\mathrm{x}}{ }_{\mathrm{x}}, \mathrm{E}_{\mathrm{y}}^{\prime}\right)$ are the coordinates of the aggregator $\mathrm{E}$ relative to the base station. The double $\left(\mathrm{A}^{\prime}{ }_{\mathrm{x}}, \mathrm{A}^{\prime}{ }_{\mathrm{y}}\right)$ is the coordinates of node A relative to the frame of reference of the base station. There is no problem having the base station compute all node coordinates in its own frame of reference and store all node positions in both the aggregator relative frame of reference and in the base station's own frame of reference because the base station is a full powered mains connected PC with large memory, disk capacity and fast CPU. To deal with networks containing slow moving nodes, this whole process of network localization as described above needs to be scheduled to be repeated at regular intervals.

\section{DISCUSSION}

It is worth now considering the relative costs of this approach to localization. For the localization scheme described here we need just 3 simple omnidirectional antennas with an amplifier and one $\mathrm{ADC}$ (analog to digital converter) chip. The ADC chip is multiplexed between the 3 antennas so that one rather than $3 \mathrm{ADC}$ chips is required per detector/localizator. The antennas and amplifier are of negligible cost compared with the ADC chip. Therefore in this design we only need to pay for one ADC chip per subnet (since only the aggregator node requires an RSSI detector) and therefore $\mathrm{N} 1+1$ ADC chips in total. By comparison in other schemes, every node and the base station has a detector so requiring considerably more, namely $3 \mathrm{~N} 1 \mathrm{~N} 2+3$, ADC chips without multiplexing. Since battery life is an essential consideration in any WSN, we must also look at the costs of on-board voltmeters for providing the battery level to the software of every node. The digital voltmeter is essentially an ADC chip and so it adds to the cost of the node. The ADC chip like other circuitry takes low power to run but also in sampling the input analog signal to convert it to a digital signal the ADC consumes power from this input signal. In the case of taking power from the antenna signal as input for a localizator this is negligible but in the case of an inbuilt battery level meter the ADC is continually drawing power from and thus draining the battery. Thus the ADC as a voltmeter continuously runs down the node's battery (as with mobile phones needing regular recharging even if not used). Battery operated computers regularly come with battery level meters inbuilt as standard and so the recommendation here for extending the

working life of a WSN is to disable or remove this part of the hardware for the construction of all nodes. Additionally digital voltmeters show hysteresis so that the State of Charge is not accurate until long periods of use after which the batteries have also been run down. Also note that when a 
node is receiving a signal from another node it does not need an ADC chip for this because the signal is binary rather than having an arbitrary amplitude as in an analog signal. Thus it is recommended here to remove all ADCs used for batteries in WSN nodes and only use one ADC chip per localizator and only on the base station and on all aggregator nodes.

We can also optimize the WSN by adjusting the initial signal strength $\psi_{0}$ if the nodes allow this flexibility. There is no need to waste energy with $\psi_{0}$ initially higher than necessary for the given size of the WSN. The minimum necessary value of $\psi_{0}$ for communication over a distance $r$ is $\psi_{m}=r^{2} \Delta \psi$ so we only need $\psi_{0}>\psi_{m}$. If $\psi_{0}$ attenuates during operation due to battery depletion then controlling $\psi_{0}$ could extend the longevity of the WSN. Since distance measurement depends crucially on knowing $\psi_{0}$ and there is the possibility of $\psi_{0}$ attenuating due to battery depletion we adopt the following assumptions. Firstly the transmission hardware attempts to send signals of the same constant intensity at the source $\psi_{0}$ so far as the battery level permits. Secondly if the WSM has non-moving nodes then the distances between nodes need only be measure once at the start of the WSN: the localization process is only required at the start-up of the WSN. Thirdly if the WSM has moving nodes or $\psi_{0}$ is different for each node, then each node needs a localizator to read its own local signal strength $\psi_{0}$ and then it must broadcast this value so that other nodes will make correct distance measurements.

A dense WSN can be defined as one where full connectivity is initially possible. If the whole WSN fits into a circle of radius $\mathrm{R}$ and $R<\rho$ then we have a dense WSN. The dense WSNs have the advantage that the architecture and connectedness of the WSN can be dynamically changed: nodes can be moved in and out of clusters as desired. So when a node has failed a replacement can be swapped in to the subnet. With such density it is possible to have many nodes dormant (an perhaps recharging with attached solar cells) and only becoming active when a cluster head has removed a sensor node from the cluster because its radio radius $\rho$ has dropped below the distance between the sensor and its aggregator node: roughly $\rho<R / \sqrt{N_{1} N_{2}}$. Even when $\psi_{0}$ for a node has dropped below the critical $\Delta \psi$ value there is still residual battery life left in its battery and this could be used to light a flashing LED on the node whose rate of flashing slows down to zero as the battery dies. In removing a dying sensor node from its cluster, the aggregator should shut down all its functions and leave the battery only driving the flashing LED. The flashing LED immediately lets the network manager know exactly which sensor node needs a battery replacement.

\section{CONCLUSIONS}

Rather than using directional antennas to determine angles, the cheaper and simpler omnidirectional antennas can be used for network localization provided each localizator has at least 3 omnidirectional antennas in manufacturer fixed positions relative to the node. The antennas should be spaced evenly at the corners of an equilateral triangle around the node which will detect the relative locations of other nodes. The equations for the position $\left(\mathrm{x}_{\mathrm{S}}, \mathrm{y}_{\mathrm{S}}\right)$ of a radio source are linear and therefore quick to compute. The formulas for computing $\left(\mathrm{x}_{\mathrm{S}}, \mathrm{y}_{\mathrm{S}}\right)$ can also be put into the hardware circuitry for the localizator to speed up the localization process and reduce the computational burden and energy consumption of a battery-based node. Alternatively the aggregators can send their raw data $\left\{\psi_{1}, \psi_{2}, \psi_{3}\right\}$ to the base station and the calculation of $\left(\mathrm{x}_{\mathrm{S}}, \mathrm{y}_{\mathrm{S}}\right)$, the coordinates of a node relative to an aggregator and the calculation of $\left(\mathrm{x}_{\mathrm{s}}{ }_{\mathrm{p}} \mathrm{y}_{\mathrm{s}}{ }_{\mathrm{s}}\right)$, the coordinates of that same node relative to the base station, are then all done on the more powerful base station 
PC. Note that not all nodes in the WSN need a localizator: only the base station and aggregator nodes require a localizator thereby reducing equipment costs. However if nodes in the WSN can move freely or is ad hoc, or if node radio signals are subject to considerable attenuation as the battery discharges, then all nodes should have localizators. Furthermore the ADCs for battery level readings should be disabled or removed from the nodes. This reduces the cost of the WSN considerably. Further savings in the battery life of the aggregators can be achieved by less frequent execution of the localization process when nodes are slow moving or static and optimizing $\psi_{0}$ for all nodes also saves battery life.

\section{REFERENCES}

[1] N Alrajeh, M Bashir, B Shams, "Localization Techniques in Wireless Sensor Networks", International Journal of Distributed Sensor Networks, vol 2015, art 304628, 9 pp,2013 Hindawi Publishing Corporation.

[2] J Rankin, "Map Making From Tables", International Journal of Computer Graphics and Animation, April 2013, Vol 3, Nr 2.

[3] M Nilsson, "Directional Antennas for Wireless Sensor Networks", Martin Nilsson. SICS, P.O.B.1263, SE-164 29 Kista, Sweden from DOT adhoc09 AT drnil DOT com, 2009.

[4] L Mottola, T Voigt, G Picco, "Electronically-switched Directional Antennas for Wireless Sensor Networks: A Full-Stack Evaluation", Sensor, Mesh and Ad Hoc Communications and Networks (SECON), 2013 10th Annual IEEE Communications Society Conference, June 2013.

[5] G Tarter, L Mottola, G Picco, "Directional Antennas for Convergecast in Wireless Sensor Networks: Are They A Good Idea?”, University of Trento Italy, http://disi.unitn.it/ picco/papers/mass 16.pdf

[6] G Whyte, "Antennas for Wireless Sensor Network Applications", PhD thesis, Department of Electrical and Electronic Engineering, University of Glasgow, 2008.

[7] Q Wang, H Dai, Z Zheng, M Imran, A Vasilakos, "On Connectivity of Wireless Sensor Networks with Directional Antennas”, Sensors Journal, 17, 134, 22pp, 2017.

[8] J Rankin "Optimal Data Collection for a 2-Level Balanced WSN", International Journal of Wireless and Mobile Networks, Vol 9, No 3, 2017.

\section{AUTHOR}

Dr J Rankin has undertaken research over 45 years starting with General Relativity and Mathematical Physics at the University of Adelaide followed by Computer Graphics, Fuzzy Logic Systems and Games Technology at La Trobe University and most recently Wireless Sensor Networks with Charles Sturt University Study Group Melbourne.

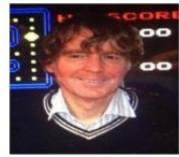

